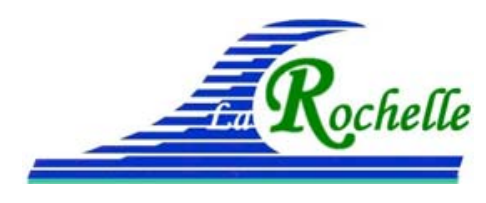

XV'̀mes Journées Nationales Génie Côtier-Génie Civil

La Rochelle, 29 au 31 mai 2018

DOI:10.5150/jngcgc.2018.003 @ Editions Paralia CFL

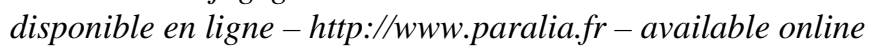

\title{
Influence du débit fluvial sur les fluctuations de niveau d'eau et de courant dans la Garonne tidale près de Bordeaux
}

\author{
Alexis BEUDIN ${ }^{1}$, Aldo SOTTOLICHIO ${ }^{1}$ \\ 1. Université de Bordeaux, UMR CNRS 5805 EPOC - OASU, Bâtiment B18 Allée \\ Geoffroy Saint-Hilaire, CS 500253, 33615 Pessac CEDEX, France. \\ alexis.beudin@u-bordeaux.fr; aldo.sottolichio@u-bordeaux.fr
}

\section{Résumé :}

L'étude porte sur l'évolution de la marée dans la portion fluviale de l'estuaire de la Gironde, à partir de l'analyse d'une chronique de mesures de courant à Bordeaux longue de plus de dix ans. L'analyse harmonique révèle que l'amplitude de la principale composante de marée semi-diurne (M2) diminue avec l'augmentation du débit fluvial, tandis que le niveau moyen de la surface libre est surélevé et les courants résiduels dirigés vers l'aval. Les fluctuations du débit fluvial modifient le rapport d'amplitude et la différence de phase entre les composantes harmoniques M2 et M4, modifiant l'asymétrie du signal de marée ; mais n'affectent pas la phase relative entre les signaux de hauteur d'eau et de courant caractéristique de l'estuaire. Les fluctuations du débit fluvial ne sont pas les seuls facteurs responsables de l'évolution pluriannuelle de la marée en amont de l'estuaire.

\section{Mots-clés :}

Estuaire, Marée, Débit fluvial, Courants, Analyse harmonique.

\section{Introduction}

Les zones géographiques les plus peuplées sont généralement observées à proximité des grands estuaires. La compréhension des mouvements des masses d'eau et des sédiments à cette interface fleuve-terre-mer est indispensable pour appréhender les risques liés au développement urbain et industrialo-portuaire (inondation, pollution) ou pour évaluer le potentiel énergétique des courants. L'objectif de cette étude est de mieux comprendre l'influence du débit fluvial sur l'hydrodynamique dans la portion amont des estuaires afin d'améliorer les prévisions de marée pour les gestionnaires et acteurs économiques de la zone.

\section{Matériels et méthodes}

\subsection{Site d'étude}

Bordeaux est situé dans la section tidale du fleuve Garonne, à environ $95 \mathrm{~km}$ en amont de l'embouchure de l'estuaire de la Gironde (figure 1). La marée est principalement semi-diurne et le marnage est compris entre 3 et 6 m (ROSS \& SOTTOLICHIO, 2016). 


\section{Thème 1 - Hydrodynamique côtière}

L'onde de marée est déformée par rapport à l'embouchure, de sorte que la période de flot est plus courte que la période de jusant. Le débit fluvial moyen annuel est d'environ $500 \mathrm{~m}^{3} / \mathrm{s}$ et peut varier entre des valeurs journalières de $100 \mathrm{~m}^{3} / \mathrm{s}$ en période d'étiage à $5000 \mathrm{~m}^{3} / \mathrm{s}$ en période de crue.

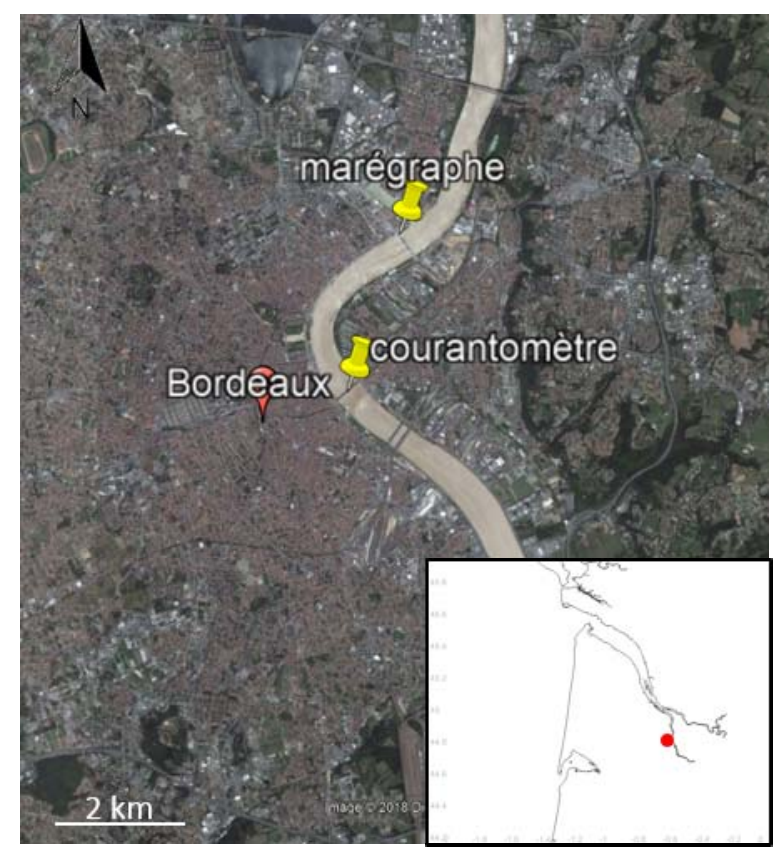

Figure 1. Position des instruments acoustiques mesurant les fluctuations de la hauteur d'eau et les courants dans la Garonne à Bordeaux.

\subsection{Collection et analyse de données}

Des mesures de courant dans l'axe du chenal ont été réalisées par le port de Bordeaux (GPMB) avec un profileur de courant acoustique horizontal à 5 cellules (ADCP Nortek AWAK $1 \mathrm{MHz}$ ) placé juste sous le niveau des plus basses mers au droit de l'arche

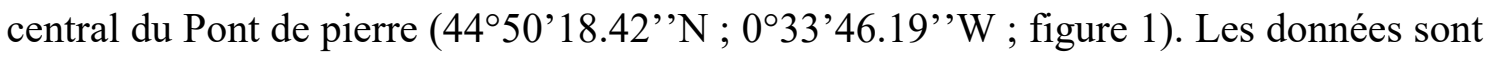
disponibles de septembre 2004 à avril 2016, à un intervalle d'une minute. Ces données ont été moyennées spatialement (sur les 5 cellules du profileur de courant), pour se focaliser sur les évolutions temporelles du signal. En complément, les évolutions de la hauteur d'eau sont enregistrées toutes les 5 min au marégraphe du port de Bordeaux, situé à moins de $3 \mathrm{~km}$ en aval du courantomètre $\left(44^{\circ} 51^{\prime} 35.89^{\prime}{ }^{\prime} \mathrm{N} ; 0^{\circ} 33^{\prime} 10.06^{\prime}\right.$ ' $\mathrm{W}$; figure 1). Les débits journaliers (Qj) de la Garonne à Tonneins, situé $50 \mathrm{~km}$ en amont de la limite d'influence de la marée, sont fournis par la DREAL Midi-Pyrénées via la Banque Hydro (http://www.hydro.eaufrance.fr/).

Une analyse harmonique avec T_TIDE (PAWLOWICZ et al., 2002; ARETXABALETA et al., 2017) a été réalisée sur des intervalles annuels et mensuels afin d'estimer l'évolution de l'amplitude des principales harmoniques de marée de 2004 


\section{XVèmes Journées Nationales Génie Côtier - Génie Civil \\ La Rochelle, 29 au 31 mai 2018}

à 2016. Les variations résiduelles (ou subtidales) sont obtenues en appliquant un filtre passe-bas avec une coupure de $33 \mathrm{~h}$.

\section{Résultats}

Les chroniques des niveaux d'eau et des vitesses du courant à Bordeaux sont comparées à l'évolution des débits journaliers de la Garonne (figure 2). La comparaison de deux cycles morte-eau/vive-eau (avec des coefficients de marée similaires) en période de crue ou d'étiage (figure 2d-e) montre clairement les effets de l'augmentation du débit fluvial sur les niveaux d'eau et les vitesses du courant : réduction du marnage, surélévation du niveau moyen, réduction de l'amplitude des oscillations de la vitesse du courant, et résiduelle de courant négative (vers l'aval). La suite des résultats tente de préciser cette observation et quantifier son importance.

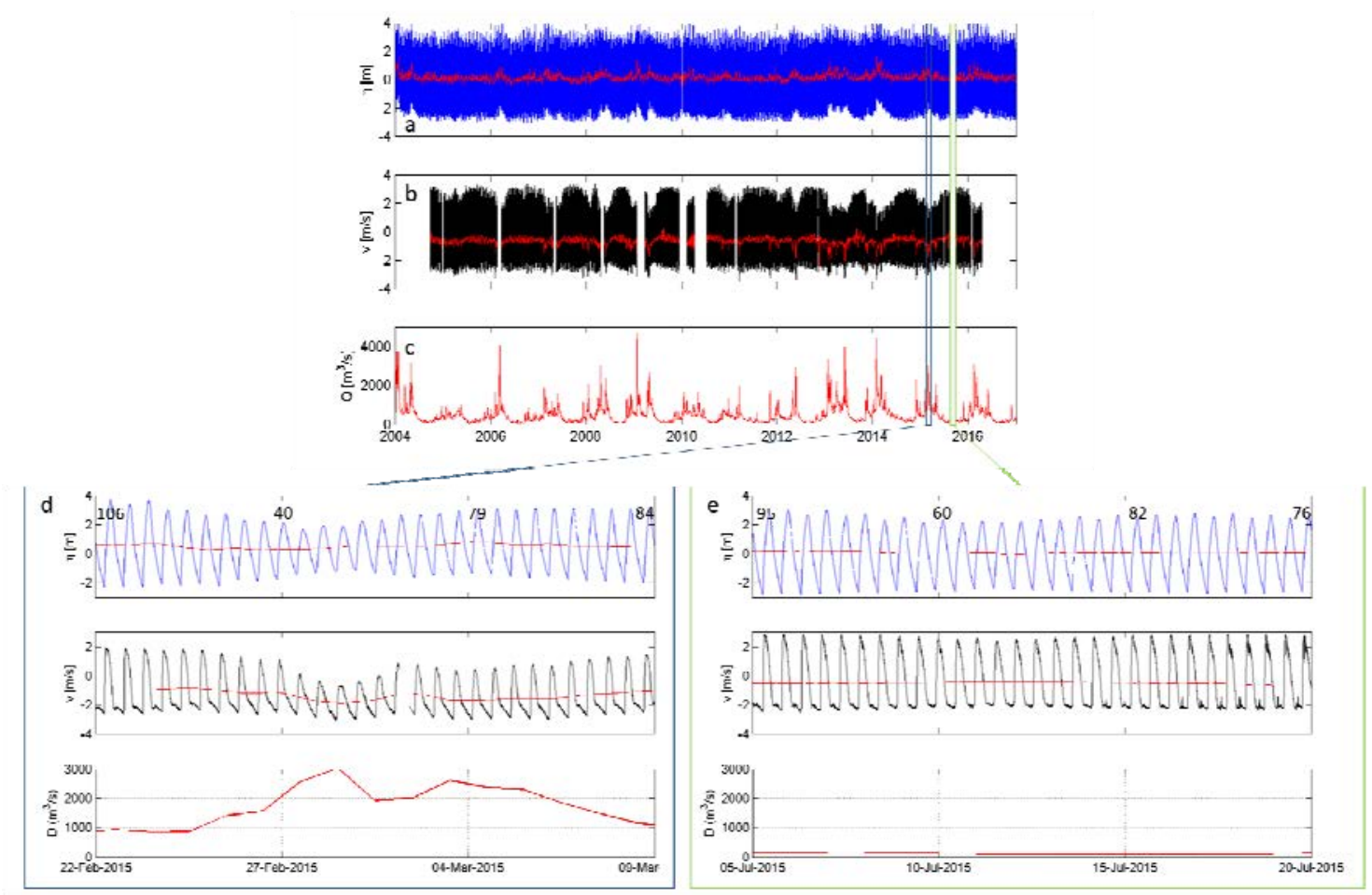

Figure 2. Chronique de (a) niveau d'eau et (b) vitesse du courant à Bordeaux, et (c) débit journalier de la Garonne en amont de 2004 à 2016. (3\% de la chronique des niveaux d'eau est inexploitable contre $27 \%$ pour les courants). La courbe rouge dans (a) et (b) correspond à la résiduel de marée. Les coefficients de marée sont indiqués tous les 5 jours sur (d) et (e).

\subsection{Composantes harmoniques et asymétrie}

La marée au niveau de Bordeaux est dominée par une variabilité semi-diurne, en particulier par la composante harmonique $\mathrm{M} 2(\mathrm{~T}=12.42 \mathrm{~h}$, a=1.96 m et $1.84 \mathrm{~m} / \mathrm{s})$. 


\section{Thème 1 - Hydrodynamique côtière}

L'amplitude de M2 décroit significativement avec l'augmentation du débit fluvial (figure 3), tandis que les fluctuations de l'amplitude de S2 (la deuxième plus importante harmonique: $\mathrm{T}=12 \mathrm{~h}, \mathrm{a}=0.52 \mathrm{~m}$ et $0.45 \mathrm{~m} / \mathrm{s}$ ) restent dans l'intervalle de confiance de la moyenne annuelle. La différence de phase entre les signaux M2 de hauteur d'eau et de courant ("phase relative") reste égale à $\sim 55^{\circ}$, typique d'une onde de marée mixte "stationnaire/progressive" (FRIEDRICHS \& AUBREY, 1994).
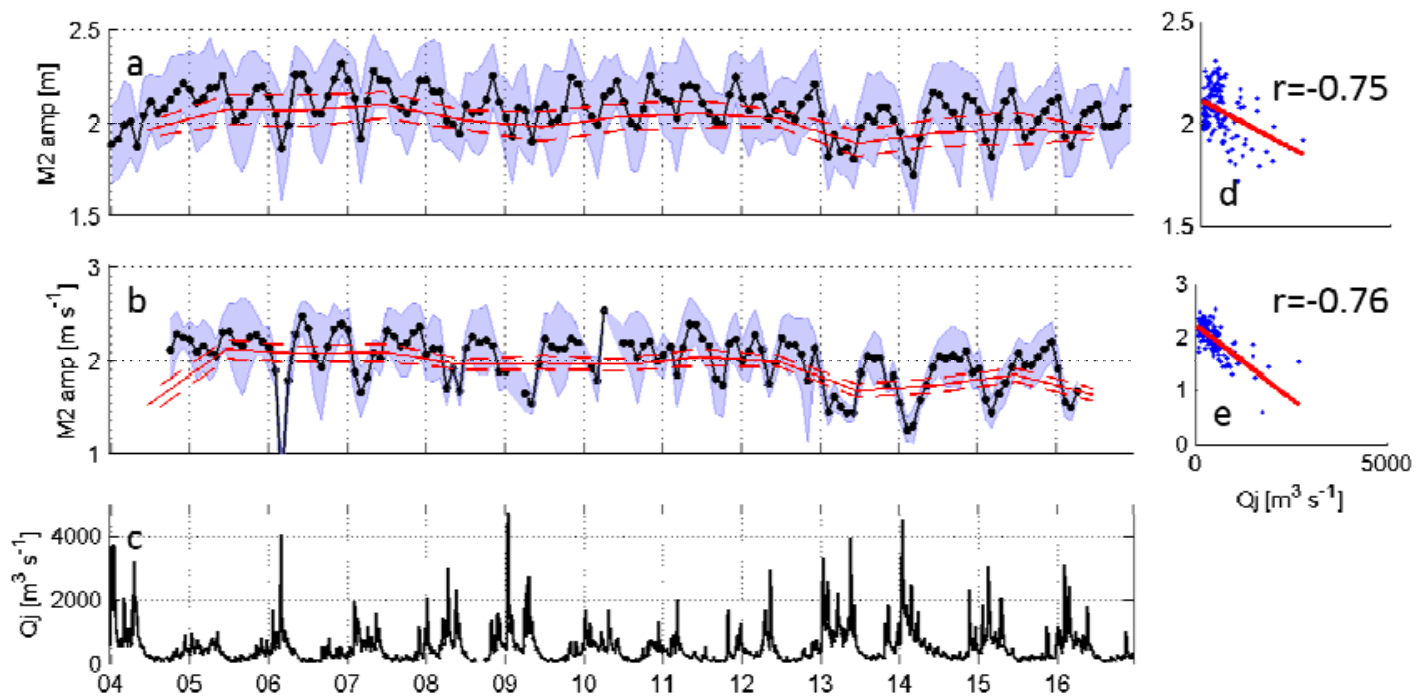

Figure 3. Évolution de l'amplitude M2 moyenne mensuelle (points noirs) du signal (a) de hauteur d'eau et (b) de courant, en parallèle (c) du débit fluvial journalier, de 2004 à 2016. Sur les figures (a) et (b), l'enveloppe bleu représente l'estimation de l'erreur de

$T \_T I D E$ (intervalle de confiance à 95\%), et les lignes rouges correspondent aux moyennes annuelles (l'erreur est en pointillé). Les résultats sur les courants en 2004 et

2016 doivent être analysés avec précaution car ces années présentent seulement 3 mois de mesures. Les quadrants (d) et (e) représentent la corrélation entre le débit fluvial et l'amplitude M2 de hauteur et de courant, respectivement.

Le rapport entre l'amplitude de la composante quart-diurne M4 (T=6.21 h) et l'amplitude de M2, caractérisant en partie l'asymétrie de la marée, augmente avec le débit fluvial (jusqu'à 25\%). La différence de phase entre les signaux M2 et M4 est réduite avec l'augmentation du débit fluvial $\left(\sim 10^{\circ}\right)$. Il en résulte un retard de l'étale de basse mer de $20 \mathrm{~min}$, et un amoindrissant du flux net dirigé vers l'amont de $30 \%$ (figure 4). 


\section{XVèmes Journées Nationales Génie Côtier - Génie Civil La Rochelle, 29 au 31 mai 2018}
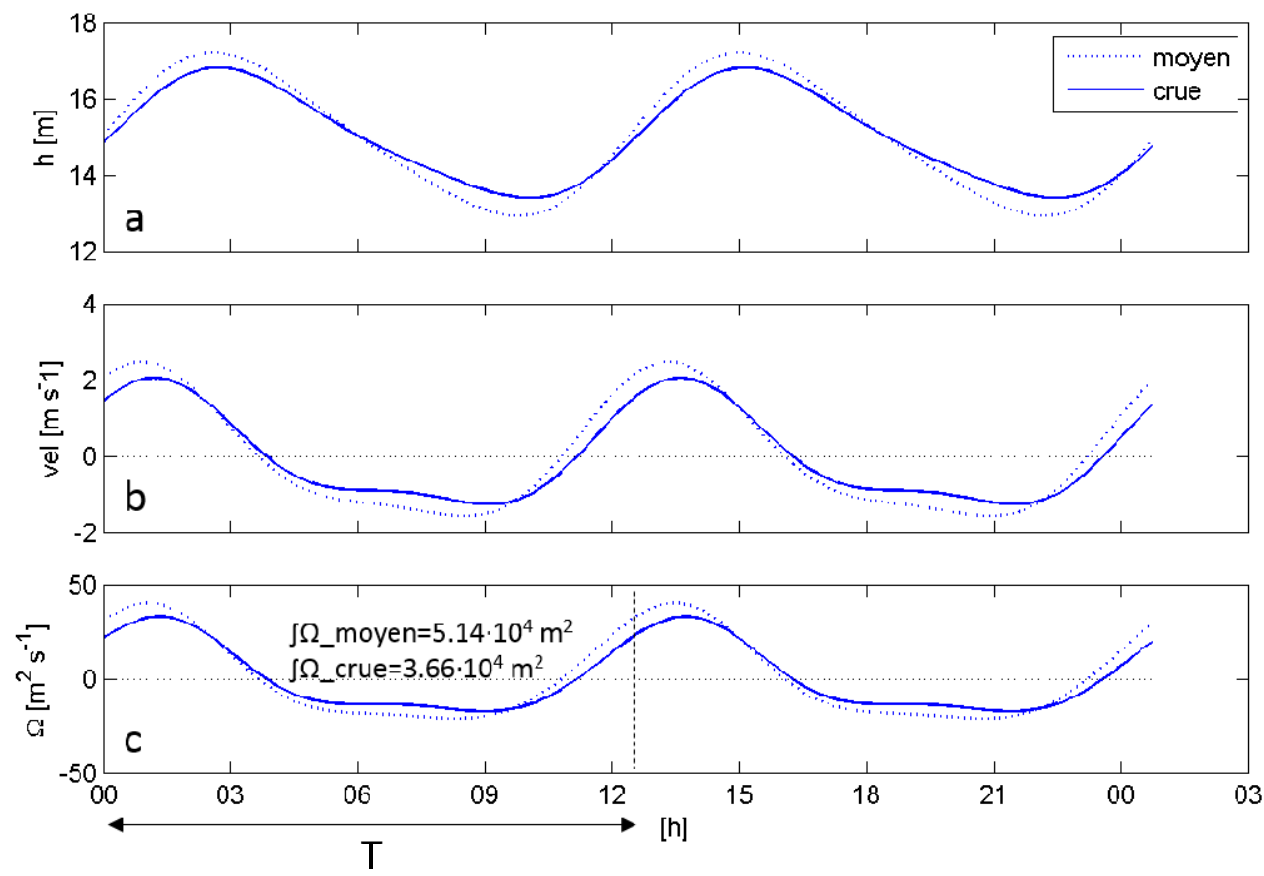

Figure 4. Reconstitution du signal de marée avec les composantes M2 et M4 en condition moyenne (pointillé) et en période de crue (celle de février 2015). Les figures (a), (b) et (c) représentent les signaux de hauteur d'eau, vitesse du courant et débit de marée (défini comme le produit hauteur fois vitesse), respectivement. Le flux net $\int \Omega$ est l'intégrale du débit de marée sur un cycle complet (12.42 h).

\subsection{Niveaux et courants résiduels}

En période d'étiage $\left(\mathrm{Qj}<500 \mathrm{~m}^{3} / \mathrm{s}\right)$ et sans compter les variations saisonnières (détectées par un filtre passe-bas avec une fréquence mensuelle), les niveaux d'eau résiduels varient de $-0.2 \mathrm{~m}$ (en morte-eau) à $0.2 \mathrm{~m}$ (en vive eau), suivant la notion de super-élévation décrite dans MEHTA (1990). En période de crue, la surcote atteint 0.5 $\mathrm{m}$ pour un débit fluvial de $1500 \mathrm{~m}^{3} / \mathrm{s}$ et $1 \mathrm{~m}$ pour un débit de $3000 \mathrm{~m}^{3} / \mathrm{s}$ (figure $2 \mathrm{a}$ ). Globalement, les fluctuations des niveaux d'eau résiduels et du débit fluvial sont bien corrélés $(\mathrm{r}=0.67$; figure $5 \mathrm{a})$.

Les courants résiduels sont généralement dirigés vers l'aval. En période d'étiage, les vitesses résiduelles sont comprises entre $0.2 \mathrm{~m} / \mathrm{s}$ (en morte-eau) et $0.8 \mathrm{~m} / \mathrm{s}$ (en viveeau). En période de crue $\left(\mathrm{Qj}>2500 \mathrm{~m}^{3} / \mathrm{s}\right)$, les vitesses résiduelles peuvent atteindre 2 $\mathrm{m} / \mathrm{s}$ (figure $2 \mathrm{~b}$ ), et la renverse de flot n'a pas lieu (figure $2 \mathrm{~d}$ ). Globalement, les fluctuations des vitesses résiduelles et du débit fluvial sont fortement corrélées ( $\mathrm{r}=$ 0.82 ; figure $5 b)$. 


\section{Thème 1 - Hydrodynamique côtière}
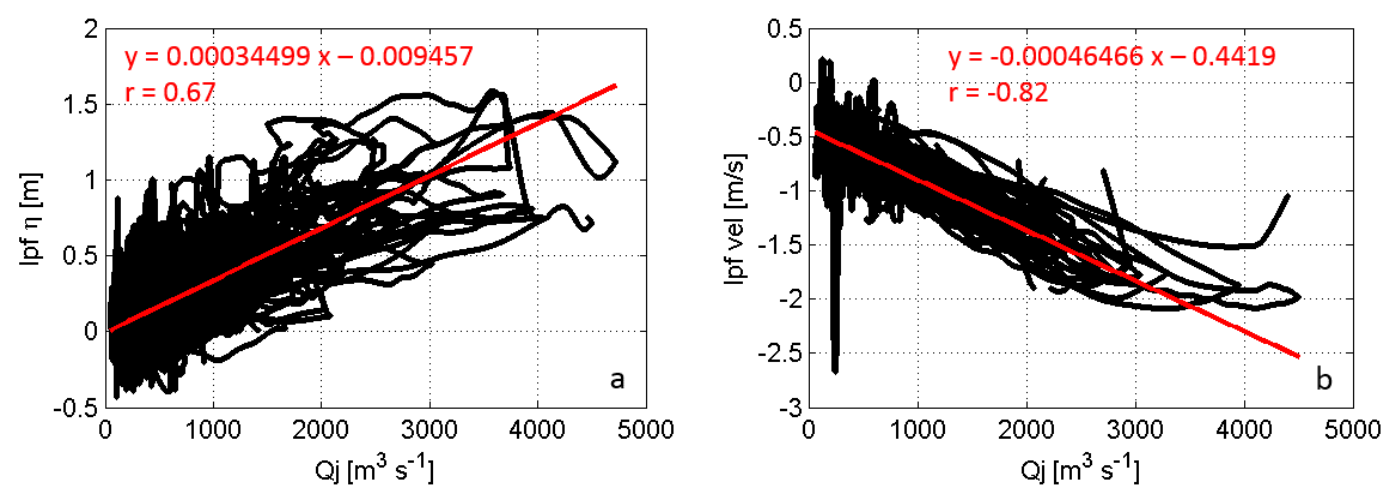

Figure 5. Relations entre le débit fluvial et les résiduelles (a) de niveau d'eau et (b) de vitesse du courant.

\section{Discussion}

\section{1 Évolution de la marée en condition d'étiage}

Outre l'influence du débit fluvial, on peut se demander si d'autres facteurs ont impacté les caractéristiques de la marée ces dix dernières années. Une analyse harmonique a été réalisée sur les périodes présentant un débit fluvial inférieur à la moyenne $(\mathrm{Qj}<500$ $\mathrm{m}^{3} / \mathrm{s}$ ). Les résultats (figure 6) montrent une diminution de l'amplitude de M2 d'environ $10 \%$ entre 2006 et 2015 (5\% entre 2008 et 2009). Les résultats de JALÓN-ROJAS (2016) indiquent que le marnage a augmenté de $15 \%$ entre 1953 et 2014 (10\% entre 1994 et 2005) en lien avec les évolutions morphologiques en aval de l'estuaire, mais qu'entre les années 2005 et 2014 contrastées en terme de débit fluvial moyen annuel (figure 2c), le marnage a augmenté en période d'étiage $\left(100<\mathrm{Qj}<250 \mathrm{~m}^{3} / \mathrm{s}\right)$ et diminué en période de crue $\left(700<\mathrm{Qj}<850 \mathrm{~m}^{3} / \mathrm{s}\right)$ en raison des différences de rugosité $\mathrm{du}$ fond. Il reste donc à étudier les évolutions morpho-sédimentaires de l'estuaire ces dix dernières années pour comprendre leur influence sur la propagation de la marée et vérifier ces résultats à l'aune de ceux de JALÓN-ROJAS (2016).

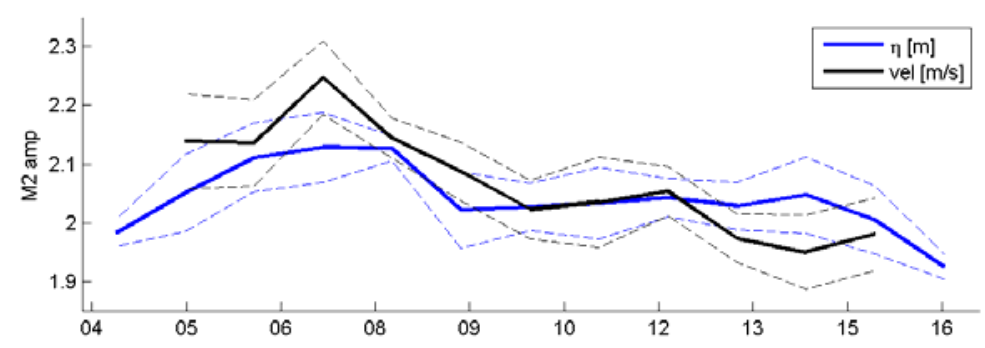

Figure 6. Évolution de l'amplitude de M2 (bleu : niveau d'eau, noir : vitesse du courant) obtenue avec T_TIDE appliqué uniquement lorsque Qj $<500 \mathrm{~m}^{3} / \mathrm{s}$ de 2004 à 2016. (Les pointillés correspondent à l'intervalle de confiance à 95\%. Les échantillons de courant en 2004 et 2016 sont insuffisants pour l'analyse harmonique.). 


\section{XVèmes Journées Nationales Génie Côtier - Génie Civil \\ La Rochelle, 29 au 31 mai 2018}

\subsection{Interactions rivière-marée}

Le taux de dissipation de l'énergie des vagues est plus important en présence d'un courant opposé au sens de propagation des vagues (THAIS et al., 2001). Par homologie, l'augmentation du débit fluvial induit un courant résiduel opposé au sens de propagation de l'onde de marée dans l'estuaire, et favorise ainsi sa dissipation. Une façon de paramétrer cet effet dans les modèles hydrodynamiques est d'ajouter un terme de frottement proportionnel au produit de la vitesse du courant de marée et de la vitesse d'écoulement fluvial (GODIN, 1991).

La présente étude considère un débit fluvial journalier. La prise en compte des fluctuations du débit fluvial à plus haute fréquence permettrait d'affiner les résultats et surtout de pouvoir étudier les feedbacks de la marée sur le débit fluvial (SASSI \& HOITINK, 2013).

\section{Conclusion}

Une analyse harmonique par portion de série temporelle a permis de monter l'influence du débit fluvial sur la marée aux échelles intra- et interannuelle (sur 10 ans) dans la portion tidale du fleuve Garonne. Elle montre l'influence particulière sur la composante semi-diurne M2. Pour les prévisions de marée en amont des estuaires, il est donc préconisé d'appliquer une modulation sur les principales composantes harmoniques en fonction du débit fluvial, plutôt qu'une correction sur le signal de hauteur d'eau prédit sans marée.

\section{Remerciements}

Ce travail a été réalisé dans le cadre du projet Gironde XL. Les auteurs remercient Alain Fort et Fabrice Klein (GPMB) pour la mise à disposition des données, et Alfredo ARETXABALETA (USGS) pour son aide avec les routines d'analyse harmonique.

\section{Références bibliographiques}

ARETXABALETA A.L., GANJU N.K., BUTMAN B., SIGNELL R.P. (2017). Observations and linear model of water level in interconnected inlay-bay system. Journal of Geophysical Research - Oceans, Vol. 122(4), pp 2760-2780. https://doi.org/10.1002/2016JC012318

FRIEDRICHS C.T., AUBREY D.G. (1994). Tidal propagation in strongly convergent channels. Journal of Geophysical Research - Oceans, Vol. 99(C2), pp 3321-3336. https://doi.org/10.1029/93JC03219

GODIN G. (1991). Compact approximations to the bottom friction term, for the study of tides propagating in channels. Continental Shelf Research, Vol. 11(7), pp 579-589. https://doi.org/10.1016/0278-4343(91)90013-V 


\section{Thème 1 - Hydrodynamique côtière}

JALÓN-ROJAS I. (2016). Évaluation des changements hydro-sédimentaires de l'estuaire de la Gironde en lien avec les pressions sur le milieu. Thèse Université de Bordeaux. Disponible en ligne sur URL : https://tel.archives-ouvertes.fr/tel-01407361

MEHTA A.J. (1990). Significance of bay superelevation in measurement of sea level change. Journal of Coastal Research, Vol. 6(4), pp 801-813. Disponible en ligne sur URL: http://www.jstor.org/stable/4297751

PAWLOWICZ R., BEARDSLEY B., LENTZ S. (2002). Classical tidal harmonic analysis including error estimates in MATLAB using T_TIDE. Computers \& Geosciences, Vol. 28(8), pp 929-937. https://doi.org/10.1016/S0098-3004(02)00013-4

ROSS L., SOTTOLICHIO A. (2016). Subtidal variability of sea level in a macrotidal and convergent estuary. Continental Shelf Research, Vol. 131, pp 28-41. https://doi.org/10.1016/j.csr.2016.11.005

SASSI M.G., HOITINK A.J.F. (2013). River flow controls on tides and tide-mean water level profiles in a tidal freshwater river. Journal of Geophysical Research - Oceans, Vol. 118, pp 4139-4151. https://doi.org/10.1002/jgrc.20297

THAIS L., CHAPALAIN G., KLOPMAN G., SIMONS R.R., THOMAS G.P. (2001). Estimates of wave decay rates in the presence of turbulent currents. Applied Ocean Research, Vol. 23(3), pp 125-137. https://doi.org/10.1016/S0141-1187(01)00014-1 